\title{
Susceptibility of the Self-Report Psychopathy Scale (SRP 4) to Response Distortion and the Utility of Including Validity Indices to Detect Deception
}

\author{
Natasha Knack, Julie Blais, J. Sebastian Baglole, and Ally Stevenson \\ Department of Psychology, Carleton University
}

Published in Psychological Assessment

\section{Author Note}

Natasha Knack (iD https://orcid.org/0000-0001-5537-3945

Julie Blais (iD https://orcid.org/0000-0001-7386-9549

J. Sebastian Baglole (D) https://orcid.org/0000-0002-4193-6249

Ally Stevenson (iD https://orcid.org/0000-0002-4615-4369

Natasha Knack is now at the School of Psychology, Victoria University of Wellington. Julie Blais is now at the Department of Psychology and Neuroscience, Dalhousie University. We have no known conflict of interest to disclose. This study was pre-registered on the Open Science Framework prior to data collection (https://osf.io/j4gmz). The dataset generated and analyzed during this study is openly available (https://osf.io/gau8f/).

Please direct correspondence to Natasha Knack, School of Psychology, Victoria University of Wellington, PO Box 600, Wellington 6140, New Zealand. Email: Natasha.Knack@vuw.ac.nz. 


\begin{abstract}
Self-report psychopathy scales are increasingly used in research and practice despite criticisms that they may be susceptible to response distortion and bias. We assessed the utility of including the Virtuous Responding (VR) and Deviant Responding (DR) validity scales from the Psychopathic Personality Inventory-Revised (PPI-R) for identifying underreporting and overreporting, respectively, on both the full and short-form versions of the Self-Report Psychopathy scale (SRP 4 and SRP-SF) in a pre/post experimental design. Using a sample of 384 male community members and a clinical comparison group of 99 males from a forensic outpatient program, we demonstrated that SRP scores were more susceptible to overreporting than underreporting, and that overreporting significantly and negatively affected convergent validity. Finally, baseline psychopathy scores were unrelated to successful response distortion (i.e., changing scores in correct direction while remaining undetected by the validity scales). It is recommended that assessments using self-report psychopathy scales consider including validity indices to detect response distortion. In doing so, it will be important to consider that general impression management may be conceptually distinct from specific forms of response distortion, such as the intentional amplification or minimization of psychopathic traits.
\end{abstract}

Keywords: psychopathy; self-report; underreporting; overreporting; response bias; deception

\title{
Public Significance Statement:
}

People can manipulate their responses on self-report scales meant to measure psychopathic traits, which may negatively affect conclusions that are made based on those responses. Self-report psychopathy scales should consider including ways of identifying people who may be intentionally responding dishonestly. 


\section{Susceptibility of the Self-Report Psychopathy Scale (SRP 4) to Response Distortion and the Utility of Including Validity Indices to Detect Deception}

Psychopathy is a personality construct associated with a combination of disordered interpersonal, affective, lifestyle, and antisocial facets (Vitacco et al., 2005). Psychopathy can be measured using clinician-rated assessments, most commonly the Psychopathy Checklist-Revised (PCL-R; Hare, 2003), as well as self-report measures. Unlike the PCL-R, self-report measures do not require trained assessors or extensive file reviews and interviews, and thus may provide a more efficient and practical tool for assessing psychopathy. However, concerns have been raised about the use of self-report measures in assessments of personality and psychopathology due to their potential susceptibility to response distortion (i.e., underreporting or overreporting certain traits for personal benefit; Bagby \& Marshall, 2003), which may impact the scale's psychometric properties (Anderson et al., 2013). These concerns may be particularly relevant for researchers and clinicians working in the field of psychopathy, given that deception and manipulation are considered core psychopathic traits (Cleckley, 1976; Gordts et al., 2017).

Despite these concerns, research is mixed on the existence of a relationship between psychopathy and response distortion (e.g., Miller et al., 2011; Ray et al., 2013), whether psychopathy scores are related to an enhanced ability to deceive on self-report measures (Book et al., 2006; Edens et al., 2000, 2001; Marion et al., 2013), and whether response distortion actually negatively affects the validity of scale scores (Watts et al., 2016). These inconsistencies could be partially explained by differences in the self-report measures and samples being studied, as well as how the authors defined and measured response distortion. Certainly, whether validity indices are actually measuring deliberate response distortion and whether self-reported scores should be adjusted based on the results of these indices is still greatly debated (McGrath et al., 2010; 
Rholing et al., 2011). However, the growing use of self-report psychopathy measures (Kelsey et al., 2015) combined with evidence that, given the right motivation, people can distort their responses on self-report scales (Anderson et al., 2013; Kelsey et al., 2015) necessitates further research into these topics.

Some self-report psychopathy measures include validity indices to detect response distortion, such as the Psychopathic Personality Inventory - Revised (PPI-R; Lilienfeld \& Widows, 2005), while others provide no means of identifying dishonest responding, such as the Self-Report Psychopathy Scale (SRP 4; Paulhus et al., 2017) and the Levenson Self-Report Psychopathy Scale (LSRP; Levenson et al., 1995). Furthermore, the SRP has often been excluded from systematic examinations of response distortion (e.g., for a meta-analysis, see Ray et al., 2013), although there is evidence that it can be susceptible to positive impression management (Kelsey et al., 2015). What effect, if any, distortions have on the psychometric properties of the SRP is still unknown. In the current study, we explored the impact of intentional response distortion on both the full and short-form versions of the SRP (version 4; Paulhus et al., 2017). We also included the Virtuous Responding (VR) and Deviant Responding (DR) validity scales from the PPI-R (Lilienfeld \& Widows, 2005) to assess the utility of including validity indices in conjunction with the SRP. Finally, we examined whether participants who successfully changed their SRP scores without being detected by the validity indices showed higher baseline levels of psychopathy than those detected as dishonest responders.

\section{Measurement of Psychopathy}

The PCL-R remains one of the most extensively validated psychopathy assessment instruments (Hare, 2003; Verschuere \& te Kaat, 2020) and involves scoring individuals on 20 items using information from an extensive file review and semi-structured interview. The 
underlying factor structure of the PCL-R is best captured by four facets representing the interpersonal, affective, behavioral, and antisocial features of psychopathy (Neumann \& Pardini, 2014; Zwets et al., 2015). As the PCL-R is both resource-intensive and requires specialized training to complete, the SRP was created as a PCL-R proxy measure (Paulhus et al., 2017), intended to provide an efficient alternative to researchers and clinicians assessing psychopathy in both forensic and community samples. More recent iterations of the SRP have improved the scale's psychometric properties, as well as expanded the number of items assessing antisocial behavior (Sellbom et al., 2018; Williams et al., 2007). Examinations of the SRP's factor structure have generally supported the same four-facet model (for a review, see Boduszek \& Debowska, 2016). A 29-item short-form version of the SRP (i.e., SRP-SF) has been found to be highly correlated with the full-length measure ( $r=.92$; Paulhus et al., 2017) and also conforms to the four-facet model (Gordts et al., 2017). While self-report scales offer an attractive alternative to intensive clinical assessments, one of the biggest concerns remains their susceptibility to possible response distortion.

\section{Psychopathy and Response Distortion}

There are several ways in which people can engage in response distortion. Underreporting, also referred to as positive impression management or "faking good", involves intentionally portraying oneself in an overly favorable light in order to conceal negative traits or behaviors. Overreporting, also known as malingering or "faking bad", involves the intentional exaggeration of psychiatric or psychopathological traits/symptoms (Ray et al., 2013; Uziel, 2010). People in both forensic and non-forensic populations may engage in deliberate response distortion for a variety of reasons. Forensic samples, for example, may underreport negative traits/symptoms to avoid harsher punishments and higher risk classifications; alternatively, they 
may overreport these negative aspects in an attempt to mitigate criminal responsibility (Sellbom, 2017; Watts et al., 2016). Community members may present themselves in an overly positive light during a job interview, or exaggerate negative symptomology to increase an insurance settlement (Bagby \& Marshall, 2003). In both cases, the motivation is key; only intentional (or experimentally induced) response distortion is expected to affect criterion validity (Holden, 2008; Hough, 1998).

Despite concerns that people with psychopathic traits may engage in more deliberate response distortion, several studies have found only negligible relationships between psychopathy and underreporting or overreporting (Miller et al., 2011; Ray et al., 2013). Similarly, several studies have not supported the contention that psychopathy is related to an enhanced ability to respond dishonestly without detection (Edens et al., 2000; MacNeil \& Holden, 2006; Marion et al., 2013). What does seem to exist, however, is evidence that selfreport scales are susceptible to response distortion in experimental studies where participants are specifically instructed to engage in dishonest responding. Kelsey et al. (2015) used a forensic sample and a within-subjects design to explore the effect of positive impression management on three self-report psychopathy measures (i.e., SRP, LSRP, PPI-R). They also examined the efficacy of the VR scale and the Social Desirability-Psychopathy scale - originally created by Rogers et al. (2002) to distinguish between suppressed psychopathy and more general positive impression management - for detecting response distortion. Results indicated that participants were able to reduce their psychopathy scores to an average below that found in community samples (see Paulhus et al., 2017 for comparison). While both validity scales showed promise in identifying positive impression management, the VR scale had higher specificity, while the 
Social Desirability-Psychopathy scale had higher sensitivity, indicating that the latter was particularly useful at detecting suppressed psychopathy.

Anderson et al. (2013) used an undergraduate sample and a between-subjects design to explore the impact of experimentally-induced response distortion on PPI-R scores and the efficacy of the PPI-R's validity scales (VR and DR) in detecting deception. Results indicated that PPI-R scores were susceptible to both underreporting and overreporting, which in turn negatively affected the convergent validity (i.e., attenuated correlations between PPI-R and LSRP scores). The VR and DR scales demonstrated acceptable sensitivity and specificity for identifying response distortion, although classification accuracy was better for the DR scale. The authors concluded that without the use of validity scales to identify response distortion, interpretations of the resulting PPI-R scores could be quite misleading. Contrary to this conclusion, however, Watts et al. (2016) utilized a large forensic sample and demonstrated almost no effect of response distortion on the relationship between several self-report psychopathy scales (e.g., PPI, LSRP) and relevant external criteria (e.g., convergence with the PCL-R; antisocial behavior). The often debated and contradictory findings above indicate the need for continued empirical investigation of both the impact of response distortion on self-report measures of psychopathy and the implications of including validity scales in conjunction with self-report instruments.

\section{Current Study}

Building on studies by Anderson et al. (2013) and Kelsey et al. (2015), we used a mixed design to examine the susceptibility of both the full and short-form versions of the SRP to experimentally-induced response distortion (both under- and overreporting), as well as the effect of intentional response distortion on convergent validity. We also supplemented the SRP with the items from the PPI-R's validity indices to assess their efficacy in identifying response distortion 
and to explore if differences in baseline psychopathy were associated with the ability to engage in successful response distortion (i.e., changing SRP score in appropriate direction) without detection. Since Anderson et al.’s study was limited by the use of a predominately female undergraduate sample, we restricted participation to males, who typically show higher average scores of psychopathy than females (e.g., Miller et al., 2011), and recruited a community rather than student sample, as psychopathy research is sometimes criticized as being overly dependent on the latter. As with Anderson et al., we included a forensic-psychiatric comparison group when examining DR scores.

The following hypotheses and data analyses were pre-registered on the Open Science Framework prior to data collection and can be accessed here: https://osf.io/j4gmz.

\section{Research Questions and Hypotheses}

\section{Question 1}

Are the SRP total and facet scores (i.e., Interpersonal Manipulation, Callous Affect, Erratic Lifestyle, Antisocial Behavior) susceptible to response distortion among a community sample of adult men?

Hypothesis 1. Based on past research in which people have successfully changed their scores on self-report psychopathy measures in response to different instructions (Anderson et al., 2013; Kelsey et al., 2015; Rogers et al., 2002), we expected that participants in the under- and overreporting conditions would be able to significantly change (i.e., decrease or increase, respectively) their total and facet scores on the SRP from Time 1 (T1; pre-scenario) to Time 2 (T2; post-scenario). We did not expect to find significant differences in the control condition.

\section{Question 2}


How does response distortion (i.e., underreporting or overreporting) affect the psychometric validity of SRP total and facet scores?

Hypothesis 2. Given that we adopted an experimental design similar to Anderson et al. (2013), we also expected response distortions to affect convergent validity analyses. We expected that the magnitude of correlations between LSRP total and factor scores (i.e., Egocentricity, Callousness, Antisociality) completed at T1 and SRP total and facet scores completed at T2 would differ significantly between conditions.

\section{Question 3}

Can the validity indices (i.e., VR and DR scales) successfully identify participants engaging in response distortion on the SRP?

Hypothesis 3. Consistent with past research on the utility of the VR and DR scales (Anderson et al., 2013; Lilienfeld \& Widows, 2005), we expected to find significant differences in VR and DR scores at T2 between the conditions. Compared to the control condition, participants in the underreporting condition were expected to have significantly higher VR scores. Compared to the control and clinical conditions, participants in the overreporting condition were expected to have significantly higher DR scores.

\section{Question 4}

Is the ability to engage in response distortion on the SRP, without being detected by the validity indices, related to higher levels of subclinical psychopathy?

Hypothesis 4. Given that deception and manipulation are considered core psychopathic traits (Gordts et al., 2017), and that evidence exists for the relationship between psychopathy and an enhanced ability to deceive (Book et al., 2006; Gillard \& Rogers, 2015), we expected that, among participants who successfully changed their SRP total score (i.e., significant change in the 
correct direction), those with higher scores on the Interpersonal/Affective subscales (i.e., SRP Facets 1 and 2; LSRP Factors 1 and 2) at T1 would be less likely to be detected by the validity indices than participants with lower scores on these subscales.

\section{Method}

\section{Participants}

\section{Power Analysis}

We used G*Power 3.1.9.4 (Faul et al., 2007) to determine the required sample size needed to detect significant effects using an alpha of .05 and 80\% power. Based on past research (see Anderson et al., 2013; Rogers et al., 2002), we estimated small (Cohen's $d=0.25$ ) to medium (Cohen's $q=0.35$ ) effect sizes and determined that the required sample size for our proposed analyses ranged from $N=36$ for a within-participant effect in the mixed-design analysis of variance (ANOVA) to $N=405$ for the Fisher's $z$-test for independent correlations. As such, we recruited a sample of $N=420$ community respondents (see pre-registration for a more detailed power analysis).

\section{Community Members}

Participants were recruited from the panels maintained by Qualtrics. After Qualtrics project managers had excluded respondents who failed to meet certain criteria (i.e., failed attention check, non-completers, "straight liners"), we received data for 420 respondents $(n=$ 140 per condition). Inclusion criteria required participants to be citizens of Canada or the US, be at least 18 years of age, and identify as a man. After eliminating respondents based on the data cleaning plan described in our pre-registration and outlined below $(n=29)$, and removing notable outliers $(n=7)$, a total of 384 community respondents remained. Participants ranged in age from 19 to 86 years, with a mean age of $53(S D=15.1)$. The majority of participants were 
Caucasian $(85 \%)$, had secondary $(36 \%)$ or post-secondary $(61 \%)$ education, and were either employed (46\%) or retired (39\%). Most had never been convicted of a criminal offense (77\%).

\section{Forensic Psychiatric Outpatients}

Following from Anderson et al.’s (2013) suggestion that a forensic psychiatric sample would constitute a more appropriate comparison group when examining DR scores in respondents asked to overreport psychopathology, we included data from a previous study in which 100 male outpatients from a forensic psychiatric program were asked to complete the PPI$\mathrm{R}$ under standard instructions ( $n=99$ after removing one notable outlier). For the current study, we were only interested in their scores on the VR and DR subscales of the PPI-R. The original study and the secondary use of this data received ethical approval from the Research Ethics Board at the Institute for Mental Health Research. Participants in the clinical sample ranged from 21 to 75 years of age, with a mean age of $46(S D=14.0)$; the majority were Caucasian $(91 \%)$, had secondary $(29 \%)$ or post-secondary $(51 \%)$ education, and had previously been convicted of a criminal offense $(87 \%)$.

\section{Data Cleaning}

Of the 420 respondents in the community sample, 29 were removed for reporting less than $40 \%$ effort or adoption of response style (or failing to answer these items). Among the remaining community participants, $88 \%$ had complete data at $\mathrm{T} 1$ and $92 \%$ at $\mathrm{T} 2$. Those with missing data were found to be similarly distributed between the control (34.3\%), underreporting (34.3\%), and overreporting (31.4\%) conditions, and there was a maximum of three missing responses for any individual scale item. All of the clinical participants had complete data.

Newman (2014, p. 392) recommended that for construct-level analyses, "if a participant responds to any items (even a single item) from a multi-item scale, then the participant's average 
response across the item(s) answered should be used to represent the participant's scale/construct score”. Parent (2013) also advocated for this method of handling missing data, which they refer to as available item analysis, and conducted multiple simulations with both artificial and realworld datasets to compare this strategy to multiple imputation - a more complex method of handling missing data. They found that with small amounts of missing item-level data on multiitem scales, there were few differences between these two approaches, and any differences that did arise were considered to be negligible. Since only two participants had more than $20 \%$ missing data on any scale - with both missing one of four items on LSRP F2 (Callousness) - we chose not to eliminate any participants based on incomplete data, and used available item analysis to address the small amount of item-level missingness. While still imperfect, this strategy is preferable to listwise deletion and single imputation, which are commonly used but typically not recommend (Newman, 2014).

Based on Parent's (2013) recommendations, we have provided additional details about missing data rates for each scale by data point (i.e., $n \times k$, where $k=$ number of scale items). For the LSRP, there were 13 missing data points out of 7,296 (.002\%); of the 19 items, one person missed three and 10 people missed one. For the SRP, there were 32 missing data points out of 24,576 at T1 (.001\%) and 29 missing data points of out 24,576 at T2 (.001\%). Of the 64 SRP items at T1, five people missed two and 33 people missed one; at T2, one person missed four, four people missed two, and 17 people missed one. There were five missing data points out of $4,992(.001 \%)$ for the VR and five missing data points out of $3,840(.001 \%)$ for the DR; no participant was missing more than one item.

\section{Data Collection}

\section{Measures}


Brief Demographic Questionnaire. Participants were asked their age, ethnicity, level of education, employment status, and whether they had ever been convicted of a criminal offense. Employment status was not available for the clinical sample.

Self-Report Psychopathy Scale (SRP 4; Paulhus et al., 2017). The SRP is a 64-item self-report measure used to assess psychopathy in both forensic and non-forensic populations. It uses Likert responses ranging from 1 (strongly disagree) to 5 (strongly agree). This measure uses the same two-factor, four-facet model as the PCL-R (Hare, 2003). These facets include Interpersonal Manipulation (IPM), Callous Affect (CA), Erratic Lifestyle (EL), and Antisocial Behavior (ASB). The 29-item short-form version of the SRP (i.e., SRP-SF) supports a similar factor structure and both versions have demonstrated remarkably similar psychometric properties in terms of internal consistency ( $\alpha=.90$ vs. $.84-.85$, respectively), and both relative and absolute fit indices (CFI $=.84-.94$ vs. $.90-.94$ and RMSEA $=.04-.05$ vs. $.04-.09$, respectively; Declerq et al., 2015; Dotterer et al., 2017; Gordts et al., 2017; Mahmut et al., 2011; Neumann et al., 2015).

In the current study, we calculated each participant's mean scores (possible range $=1$ to 5) for the total measure and all four subscales on both the SRP and SRP-SF. For the full SRP, Cronbach's alphas ${ }^{1}$ were high at both $\mathrm{T} 1$ and T2 for the total score $(\alpha=.91$ and .96 , respectively) and ranged from moderate to high across all four facets (T1: .50 to .83; T2: .69 to .92).

Compared to the full version, the items on the SRP-SF had similarly high Cronbach's alphas at T1 and T2 for the total score ( $\alpha=.91$ and .96 , respectively), and higher alphas for all facet scores (T1: .72 to .79; T2: .86 to .89). Only community participants completed this measure.

\footnotetext{
${ }^{1}$ Since we used available item analysis to handle missing data, we used SPSS syntax provided by Parent (2013) to calculate Cronbach's alphas, as SPSS uses listwise deletion to handle missing data when calculating this statistic.
} 
Psychopathic Personality Inventory - Revised (PPI-R; Lilienfeld \& Widows, 2005).

The PPI-R is a 154-item self-report measure of psychopathic traits, which asks participants to respond to statements about themselves using Likert scale options ranging from 1 (false) to 4 (true). For the current study, only the items from the Virtuous Responding (VR; 13 items) and Deviant Responding (DR; 10 items) validity subscales were used. The VR subscale provides an indication of whether respondents view themselves more positively or negatively, and if they perceive themselves as being "relatively free of serious personality flaws" compared to “psychologically disturbed" (Lilienfeld \& Widows, 2005, p. 18). The DR subscale is intended to detect malingering (or other forms of "faking bad"), and also helps to identify respondents who may have attempted to "sabotage" the assessment. In the current study, we calculated each participant's mean scores (possible range $=1$ to 4 ) for the VR and DR scales; only T2 scores were included in the analyses. The Cronbach's alphas were moderate to high at T1 and T2 for both the VR $(\alpha=.79, .82)$ and $\operatorname{DR}(\alpha=.73, .89)$. Both the community and clinical participants completed the VR and DR scales.

Levenson Self-Report Psychopathy Scale (LSRP; Levenson et al., 1995). The LSRP is a 26-item self-report measure that asks participants to respond to statements using Likert scale options from 1 (strongly disagree) to 4 (strongly agree). Both a two-factor (i.e., Primary and Secondary psychopathy) and three-factor (i.e., Egocentricity, Callousness, Antisociality) structure have been proposed. Unlike the two-factor structure, which showed acceptable construct validity (Salekin et al., 2014) but unacceptable model fit indices in confirmatory factor analysis (Brinkley et al., 2008), the three-factor structure was found to have adequate fit (Sellbom, 2011; Wang et al., 2018). For the current study we used the three-factor model to better align with the SRP facets. This measure was only completed at T1. We calculated each 
participant's mean scores (possible range $=1$ to 4 ) for the total measure and the three subscales. We found moderate to high Cronbach's alphas for the total score $(\alpha=.87)$, and all three factors: Egocentricity $(\alpha=.86)$, Callousness $(\alpha=.66)$, and Antisociality $(\alpha=.71)$. Only community participants completed this measure.

\section{Procedure}

Prior to data collection, this study received ethical approval from the Research Ethics Board at Carleton University. Community respondents completed the study online using Qualtrics. In addition to the incentive provided by Qualtrics (e.g., Air Miles), participants were told that if they were able to adopt the specific response style without being detected by the validity scales, they would be entered into one of three draws for $\$ 100$. In reality, all participants were included in these draws and were informed of this in the debriefing form; given this deception, participants were asked to provide secondary consent after this information was revealed. The survey comprised three phases: (1) Pre-scenario (T1); (2) Scenario; and (3) Postscenario (T2).

Pre-scenario (T1). Participants first provided basic demographic information and then completed the outcome measures (i.e., LSRP, SRP 4, PPI-R VR and DR subscales) under standard instructions (i.e., to respond honestly). Given that the SRP and VR/DR subscales have different response options, we presented the SRP items in eight blocks and the VR/DR items in four blocks; both the item presentation within blocks and the presentation of the blocks themselves was randomized. The LSRP items were randomized within a single block. The order in which the LSRP and SRP/VR/DR measures were completed was also randomized.

Scenario. Participants were then randomly assigned to one of the following conditions:

(1) Control - encouraged honest responding $(n=132)$; (2) Underreporting - encouraged 
minimizing negative traits/psychological symptoms to receive a lighter prison sentence $(n=$ 127); and (3) Overreporting - encouraged amplifying negative traits/psychological symptoms to be found not criminally responsible due to a mental disorder $(n=125)$. All three scenarios informed participants that the scale included items intended to identify dishonest responders. Full scenarios are available in Appendix A of the online supplemental materials.

Post-scenario (T2). After reading the scenarios, participants were asked to complete the SRP and VR/DR validity scales a second time (same randomized block presentation described in T1), using the specific instructions provided in their scenario (i.e., underreport, overreport, respond honestly).

\section{Results}

The dataset on which the following results are based is openly available on the Open Science Framework at https://osf.io/gau8f/ (Blais et al., 2021).

\section{Susceptibility of the SRP to Response Distortion (Hypothesis 1)}

We used a 3 (condition: control, underreport, overreport) x 2 (time: T1, T2) mixed-design ANOVA to examine the impact of response distortion on SRP total and facet scores. Participants in the overreporting condition had significantly higher SRP total scores $(M=2.6, S D=0.64)$, on average, than did participants in either the control $(M=2.2, S D=0.44)$ or underreporting conditions $(M=2.2, S D=0.48), F(2,381)=27.83, p<.0001, \eta_{\mathrm{p}}^{2}=.13$. The same was true for scores on the IPM, CA, EL, and ASB facets $\left(\eta_{\mathrm{p}}^{2}=.07, .09, .10, .13\right.$, respectively). We also found a significant main effect of time, as the average SRP total score across conditions was significantly higher at $\mathrm{T} 2, F(1,381)=28.86, p<.0001, \eta_{\mathrm{p}}^{2}=.07$, as were all four facet scores $\left(\eta_{\mathrm{p}}^{2}=.03, .08, .04, .10\right)$. The significant main effects were qualified by a significant two-way 
interaction for the SRP total, $F(2,381)=65.77, p<.0001, \eta_{\mathrm{p}}^{2}=.26$, and facet scores $\left(\eta_{\mathrm{p}}{ }^{2}=.23\right.$, $.20, .20, .25)$

To follow-up the significant two-way interaction, we used mixed-design $t$-tests to explore the simple main effects of condition (specifically the control vs. response distortion conditions) on SRP total and facet scores at T1 and T2 (see Figure 1). We used a Bonferroni correction to control for familywise error rate $(\alpha=.05 / 5=.01)$. We found no significant difference in SRP total scores at $\mathrm{T} 1$ between the control condition and either the underreporting, $t(623)=0.24, p=$ $.808, d=0.04$, or overreporting conditions, $t(623)=0.58, p=.563, d=0.09$. We also found no significant difference in SRP total scores between the control condition and the underreporting condition at $\mathrm{T} 2, t(623)=0.66, p=.508, d=0.09$. In contrast, the overreporting condition had significantly higher SRP total scores at T2 than the control group, $t(623)=10.39, p<.0001, d=$ 1.05. We found similar results for all four facet scores (see Table 1).

[Figure 1 about here]

[Table 1 about here]

In order to examine the within-condition effects from $\mathrm{T} 1$ to $\mathrm{T} 2$ for each randomly assigned condition, we conducted a series of paired-samples $t$-tests for the SRP total and facet scores. We used a Bonferroni correction to control for familywise error (adjusted $\alpha=.01$ ). Contrary to our expectation, the control condition significantly lowered their SRP total, $t(131)=$ $3.89, p=.0002, d=0.34,95 \%$ CI $[0.16,0.51]$, and Interpersonal Manipulation scores, $t(131)=$ $4.69, p<.0001, d=0.41,95 \% \mathrm{CI}[0.23,0.59]$ from $\mathrm{T} 1$ to $\mathrm{T} 2$. The underreporting condition showed a significant decrease in SRP total, $t(126)=3.53, p=.0006, d=0.31,95 \%$ CI $[0.14$, 0.49], Interpersonal Manipulation, $t(126)=4.27, p<.0001, d=0.38,95 \%$ CI $[0.20,0.56]$, and Erratic Lifestyle scores, $t(126)=3.54, p=.0006, d=0.31,95 \%$ CI [0.14, 0.49], from T1 to T2. 
No significant decrease was found for Callous Affect or Antisocial Behavior. In contrast, the overreporting condition significantly increased their SRP total scores from $\mathrm{T} 1$ to $\mathrm{T} 2, t(124)=$ $7.66, p<.0001, d=0.68,95 \% \mathrm{CI}[0.49,0.88]$, as well as their scores on all four facets:

Interpersonal Manipulation, $t(124)=-6.92, p<.0001, d=0.62,95 \%$ CI $[0.43,0.81]$; Callous Affect, $t(124)=-6.99, p<.0001, d=0.62,95 \%$ CI $[0.43,0.82]$; Erratic Lifestyle, $t(124)=-6.68$, $p<.0001, d=0.60,95 \%$ CI [0.41, 0.79]; and Antisocial Behavior, $t(124)=-8.19, p<.0001, d=$ $0.73,95 \% \mathrm{CI}[0.53,0.93]$. These findings suggest that participants were better able to amplify as opposed to minimize negative traits/symptoms. This provides partial support for Hypothesis 1 .

We also conducted the above analyses using only the 29 items from the SRP-SF. The only notable change in significance was for the paired-samples $t$-test, as the decrease in total score from T1 to T2 did not quite reach significance $(p=.01)$ in the underreporting condition when using the SRP-SF compared to the full SRP. See Appendix C in supplemental materials for detailed results using the SRP-SF.

\section{Response Distortion and Psychometric Validity of the SRP (Hypothesis 2)}

To examine the impact of response distortion on the convergent validity of SRP total and facet scores, we obtained correlations between LSRP scores at T1 and SRP scores at T2. Since the LSRP and SRP use slightly different factor structures, we followed Anderson et al. (2013) and correlated LSRP Factor 1 (Egocentricity) with SRP Facet 1 (Interpersonal Manipulation), LSRP Factor 2 (Callousness) with SRP Facet 2 (Callous Affect), and LSRP Factor 3 (Antisociality) with both SRP Facet 3 (Erratic Lifestyle) and SRP Facet 4 (Antisocial Behaviour). We then used Fisher's $z$-test for independent correlations to explore differences in correlation magnitudes between conditions; we used Cohen's $q$ and $95 \%$ confidence intervals to estimate effect size. 
As shown in Table 2, we found the largest correlations among the control group, with the exception of the Callousness/Callous Affect correlation, which was slightly higher in the underreporting condition. However, correlations were not significantly different in the control versus underreporting conditions for the total or facet scores. In contrast, correlations for the overreporting condition were significantly lower than the control condition for the total and all facet scores (including two negative correlations). Effect sizes ranged from medium to large $(q=$ 0.38 to 0.86 ). This suggests that the convergent validity of the SRP total and facet scores was negatively impacted by participants' attempts to amplify negative traits, but was not particularly affected by attempts to minimize negative traits. These results provide partial support for Hypothesis 2.

[Table 2 about here]

When using the SRP-SF versus the full SRP, we found only minor changes in correlation magnitudes (maximum change in $r=.06$ ) and comparisons of correlation magnitudes between conditions (maximum change in $z=.72$ ). These changes did not impact the significance of the results. See Appendix D in supplemental materials for detailed results.

\section{Efficacy of Validity Scales at Detecting Response Distortion (Hypothesis 3)}

We conducted two one-way ANOVAs to examine the impact of condition on VR and DR scores at $\mathrm{T} 2$ and found significant between-group differences for both the VR, $F(3,479)=9.20$, $p<.0001, \eta_{\mathrm{p}}^{2}=.05$, and DR subscales, $F(3,479)=54.04, p<.0001, \eta_{\mathrm{p}}{ }^{2}=.25$. We followed up these main effects using independent pairwise comparisons to explore differences on the validity indices between the control and response distortion conditions (see Table 3). We used a Bonferroni correction to control for familywise error $(\alpha=.05 / 3=.02)$. We found no significant difference between the control and underreporting conditions for either the $\operatorname{VR}(p=.084)$ or DR 
subscales $(p=.446)$. In contrast, participants in the overreporting condition had significantly lower VR scores $(p=.0005, d=0.44)$ and significantly higher DR scores $(p<.0001, d=1.07)$, compared to the control condition. We found a similar pattern of results when comparing the overreporting and clinical samples on both the VR $(p=.003, d=0.41)$ and DR subscales $(p<$ $.0001, d=1.19)$. This suggests that the validity indices were only able to detect response distortion when participants were asked to amplify, as opposed to minimize, negative traits/symptoms. These results provide partial support for Hypothesis 3.

[Table 3 about here]

\section{Baseline Psychopathy and Detection of Response Distortion (Hypothesis 4)}

Participants in the under- and overreporting conditions were considered to have successfully engaged in response distortion if they were able to decrease or increase their SRP total scores, respectively, by at least half of one standard deviation. We found that $29.8 \%$ ( $n=$ 75) of those in the response distortion conditions were able to successfully change their score, with a larger portion of the overreporting group showing successful change $(40.8 \%, n=51)$ compared to the underreporting group $(18.9 \% ; n=24)$. When using only the items from the SRP-SF, two additional participants successfully increased their score, resulting in a very slight increase in the proportion of successful participants $(30.6 \% ; n=77)$.

Among participants who successfully changed their SRP score, those whose VR or DR score at T2 was greater than or equal to the scale's cut score were considered to have been detected by the validity indices. Using classification analyses, we determined that the optimal VR and DR cut scores (i.e., highest sensitivity with highest specificity) were 3.2 and 2.2, respectively (see Appendix E in supplemental materials for classification analyses). However, even these "optimal" cut scores showed poor sensitivity - particularly for the VR scale - which 
may have impacted the subsequent results. When using these cut scores with the full SRP, 33.3\% $(n=25)$ of participants who successfully changed their SRP total score were not detected by the validity indices; this included $79.2 \%(n=19)$ of the underreporting condition and $11.8 \%(n=6)$ of the overreporting condition. When using only the items on the SRP-SF, $33.8 \%(n=26)$ of participants who successfully changed their SRP-SF total score were not detected by the validity indices; this included $79.2 \%(n=19)$ of the underreporting condition and $13.2 \%(n=7)$ of the overreporting condition.

We used independent pairwise comparisons to determine if baseline levels of psychopathy (i.e., LSRP, SRP, and SRP-SF total and facet scores at T1) differed between participants whose successful response distortion was detected versus undetected by the validity indices. Using a Bonferroni correction to control for familywise error $(\alpha=.05 / 14=.004)$, we found no significant differences in baseline LSRP total or factor scores $(p s=.110$ to $.401, d \mathrm{~s}=$ 0.21 to 0.40$),$ SRP total or facet scores $(p s=.009$ to $.157, d s=0.35$ to 0.75$)$, or SRP-SF total or facet scores ( $p \mathrm{~s}=.058$ to $.720, d \mathrm{~s}=0.09$ to 0.46 ); see Appendix $\mathrm{F}$ in online supplemental materials for full results. These findings do not support Hypothesis 4 .

\section{Discussion}

The primary goals of this study were to assess whether SRP scores were susceptible to intentional response distortion, determine the effect of response distortion on the convergent validity of scale scores, and explore the utility of using established validity indices to detect response distortion in a male community sample. We found that the SRP (both the full and shortform version) was in fact susceptible to both underreporting and overreporting, although the effect was larger for those asked to amplify as opposed to minimize negative traits. This finding could potentially reflect the relatively low base rate of psychopathic traits in the general public 
(approximately 1\%; Reidy et al., 2018), which would make it difficult for participants to further decrease their scores in the underreporting condition. However, if that were the case, we might have expected participants' SRP total scores at T1 to be even lower than those observed; the average SRP score at T1 across all three conditions was 2.3 out of 5. At T2, participants may have avoided choosing the extreme response option needed to get a minimum score (i.e., strongly disagree), thinking that the validity scales would likely identify such obvious attempts at deception. Since Kelsey et al. (2015) have suggested that even people with psychopathic traits may not be fully aware of every undesirable aspect that could be manipulated during intentional response distortion, another possible explanation is that community members may be unaware of the specific traits or symptoms associated with this personality construct. As such, participants in our study may have had difficulty knowing which traits they should attempt to minimize.

Our results did demonstrate that overreporting, but not underreporting, significantly attenuated the correlations between the SRP (full and short-form versions) and LSRP scales, for the total and all facet scores. This suggests that intentional or experimentally induced attempts to amplify, but not minimize, negative traits/symptoms have the potential to significantly decrease the convergent validity of SRP test scores and raises questions about the impact of response distortion on other self-report psychopathy measures. For example, Anderson et al. (2013) reported similar concerns with the PPI-R. To be clear, like Anderson et al. (2013), we provided only a limited test of the potential negative effects of response distortion on psychometric properties (i.e., convergent validity); larger investigations of this issue have found only negligible effects of response distortion on the psychometric properties of self-report scales (McGrath et al., 2010; Watts et al., 2016). The negative effects of response distortion also seem confined to experimental studies where respondents are specifically told to answer dishonestly. 
At the very least, this indicates that when people do in fact engage in deliberate response distortion, this distortion has the potential to affect the interpretation of scale scores, especially for those engaging in overreporting. The fact that correlations between the SRP and LSRP were unaffected by underreporting could stem from participants struggling to lower their scores in this condition for the reasons noted above (e.g., unclear which traits to minimize).

\section{Utility of Validity Indices}

While the utility of validity indices has been called into question (see McGrath et al., 2010), others argue that they are useful in clinical evaluations (Rohling et al., 2011) and when evaluating constructs where deception may be of particular concern, such as psychopathy (Anderson et al., 2013; Kelsey et al., 2015). If not for our use of the VR and DR validity scales, $100 \%$ of participants who successfully engaged in intentional response distortion on the SRP would not have been detected as dishonest responders. The fact that two-thirds of those who successfully changed their SRP scores from T1 to T2 were detected by the VR or DR scales suggests that validity indices show some promise in identifying intentional response distortion among community members; however, this finding also suggests that these particular scales may not be the ideal option, especially for the SRP.

In all analyses, the DR scale performed better than the VR scale in identifying dishonest responders. It is possible that our experimental manipulation was more effective at eliciting overreporting than underreporting, therefore affecting the ability of the validity scales to detect deception. However, it is also possible that the problem is not the manipulation but rather the validity scales themselves. More specifically, malingering seems to be a relatively straightforward response distortion (Uziel, 2010) and our results support the DR scale's ability to detect this type of deception. On the other hand, there is growing evidence that positive 
impression management scales, such as the VR, are not measuring a response style at all, but rather an adaptive feature of people's personality (see Uziel, 2010). In fact, as opposed to being indicative of deliberate and even nefarious attempts at deception, scores on scales similar to the VR have correlated with a number of adaptive personality traits (e.g., honesty-humility, conscientiousness, and agreeableness; deVries et al., 2014) and outcomes such as reduced substance use (Luhtanen \& Crocker, 2005). Positive impression management scores thus appear to describe people with traits opposite that of psychopathy (e.g., honest, sincere, caring for others, planning ahead). It would therefore make little sense to adjust self-report psychopathy scale scores based on elevated scores on measures of positive impression management (consistent with suggestions made by Watts et al., 2016).

Higher rates of classification accuracy may require the development of validity indices specifically designed for use among populations expected to have subclinical levels of psychopathy. The VR and DR scales were not designed to measure psychopathy-specific response distortion, but rather to provide an indication of participants' general impression management and response styles (Lilienfeld \& Widows, 2005). Kelsey et al.'s (2015) finding that the Social Desirability-Psychopathy index (originally by Rogers et al., 2002) outperformed the classification accuracy of the VR scale when assessing suppressed psychopathy in a forensic sample highlights the benefit of creating more specialized validity scales. In doing so, it will be important to consider that general impression management may be conceptually distinct from specific forms of response distortion, such as the intentional amplification or minimization of psychopathic traits.

\section{"Successful" Response Distortion}


As a final note on our analyses, we were unable to replicate the findings of several studies that identified relationships between successful response distortion and elevated psychopathic traits. We attribute this largely to the fact that past research has defined "successful" response distortion as the ability to either intentionally change psychopathy scores in a specified direction from T1 to T2 (e.g., Edens et al., 2001) or remain undetected by specific validity scale cut scores when asked to under- or overreport on self-report psychopathy measures (e.g., Book et al., 2006). Studies using the former design are unable to determine if participants who successfully change their psychopathy scores would be detected as biased responders if the measure included validity scales, whereas studies using the latter design provide no indication of whether participants actually effected a notable change in psychopathy scores by engaging in intentional response distortion.

We used more stringent criteria for the current study by requiring that "successful" participants (i.e., those we would expect to have the highest levels of baseline psychopathy) were able to both intentionally change their psychopathy scores from T1 to T2 (in the correct direction, by at least a set amount) and remain undetected by the validity scales (i.e., score below the optimal cut score for the VR or DR). Only one-third of participants across both response distortion conditions were able to meet these criteria. We should note that the sensitivity and specificity analyses used to determine the cut scores reached acceptable levels for the DR scale, but sensitivity rates fell below acceptable standards for the VR scale (see Appendix E in supplemental materials). We therefore have to interpret these results with some caution. Contrary to our expectation, successful and undetected participants were not found to have higher levels of baseline psychopathy than those who successfully engaged in response distortion but were detected as biased responders. Our results are more in line with those of Marion et al. (2013), 
who found no relationship between psychopathy and successful response distortion in either an undergraduate or forensic sample using validity scales from the Minnesota Multiphasic Personality Inventory (MMPI-2-RF; Ben-Porath \& Tellegen, 2008).

\section{Limitations and Future Directions}

While the current study provided a number of interesting findings, there are several limitations that must be noted. As previously stated, the validity scales of the PPI-R, especially the VR scale, may not be the most appropriate choice when assessing response distortion for the SRP. Given the conceptual issues surrounding positive impression management, utilizing scales that are actually meant to measure suppressed psychopathy, such as the Social DesirabilityPsychopathy scale (Kelsey et al., 2015; Rogers et al., 2002), are likely a better alternative. Future research should attempt to further validate this scale, as well as develop and validate an analogous measure to detect overreporting of psychopathic traits.

In terms of scale administration, since the SRP and VR/DR measures use different Likert scales, we were unable to embed the validity items directly within the SRP. Consequently, we administered the items from both measures across a series of randomized blocks. We also used only the VR/DR subscales rather than administering the complete PPI-R. Our results are thus limited by the fact that we did not use standard procedures when administering these measures.

In terms of our experimental conditions, contrary to our expectations, the control group significantly lowered their SRP scores from T1 to T2, despite being instructed to respond honestly at both timepoints. One possible explanation for this unanticipated change is the minor differences in the control group's instructions between timepoints. At T2, participants were informed that questionnaires often have ways of detecting deception, and that they would only be entered into the bonus draw if their responses were not detected as dishonest. If control 
participants had responded dishonestly at T1, this warning may have discouraged them from doing so again at T2. Alternatively, these instructions and/or the repeated administration of this scale may have led to greater attention and reflection of the items at T2. It is also possible that participants felt the need to present themselves more favourably than they had at T1, as Furnham and colleagues (Furnham, 1986; Furnham \& Henderson, 1982) have postulated that explicitly instructing participants to respond honestly could potentially evoke a bias toward social desirability. Regardless of the reason, our findings suggest that researchers interested in this topic may benefit from studying the effects of specific response instructions used in self-report measures (e.g., to respond honestly vs. dishonestly). One suggestion would be to include openended questions at the end of self-report scales to determine possible motivations for engaging in response distortion when instructed to respond honestly.

Another study limitation pertains to the fact that participants' motivation to engage in response distortion during a hypothetical legal scenario is unlikely to adequately reflect the degree of motivation experienced by those involved in actual high-stakes criminal cases. We attempted to partially mitigate this issue by informing participants that they would only be entered into the bonus draw if their responses were not detected as dishonest. However, we acknowledge that even if this strategy were successful, considerable differences in motivational strength would remain between the real and hypothetical versions of these scenarios, thereby limiting the generalizability of our results. Regarding generalizability, our findings are also limited by the lack of ethnic diversity among our participants. The fact that the majority of participants had post-secondary education and were either employed or retired also suggests a potential lack of diversity with regard to socioeconomic status. While we intentionally used an all-male sample to increase the likelihood of recruiting participants with higher levels of 
subclinical psychopathy, this design choice prevents us from generalizing our results to people of different genders.

\section{Conclusion}

Response distortion is a potential concern for all self-report psychopathy measures, particularly those that do not contain any means of identifying dishonest responding. To expand upon previous research (Anderson et al., 2013; Kelsey et al., 2015), we examined the effect of response distortion on the full-length and short-form versions of the SRP (Paulhus et al., 2017) in a community sample of men. The fact that respondents were able to intentionally distort their SRP profiles - in some cases without being detected as dishonest responders - is a critical issue, given that response distortion was found to affect the convergent validity of test scores in the overreporting condition. Although this and other studies have demonstrated that self-report psychopathy scales may be susceptible to different forms of response distortion, this does not necessarily imply that they cannot be used appropriately. It does, however, demonstrate that researchers and clinicians working in both forensic and non-forensic contexts should remain aware that intentional response distortion is not only a possibility, but one that can significantly impact the validity of test scores and any decisions ensuing from these results.

Our findings add to the already mounting evidence supporting the need for psychopathy assessments that are reliant on self-report measures to include a mechanism for detecting intentional response distortion. While the DR scale of the PPI-R showed some promise in identifying overresponding on the SRP, the poor classification accuracy of the VR scale indicates that an equivalent validity index for detecting underreporting among community samples is not yet evident. Our results are consistent with those from previous studies suggesting that the efficacy of validity indices may depend on whether they are used within the population for which 
they were originally intended. As such, we recommend the development, standardization, and implementation of validity scales specifically intended for use in self-report psychopathy measures for non-forensic populations (i.e., those expected to have subclinical levels of psychopathy). This recommendation is especially pertinent for the SRP and other self-report psychopathy measures that do not currently contain any form of validity indices. 


\section{References}

Anderson, J., Sellbom, M., Wygant, D., \& Edens, J. (2013). Examining the necessity for and utility of the Psychopathic Personality Inventory-Revised (PPI-R) validity scales. Law and Human Behavior, 37(5), 312-320. https://doi.org/10.1037/lhb0000018

Bagby, R.M. \& Marshall, M.B. (2003). Positive impression management and its influence on the revised NEO Personality Inventory: A comparison of analog and differential prevalence group designs. Psychological Assessment, 15(3), 333-339. https://doi.org/10.1037/10403590.15 .3 .333

Ben-Porath, Y. S., \& Tellegen, A. (2008). MMPI-2-RF (Minnesota Multiphasic Personality Inventory-2-Restructured Form): Manual for administration, scoring, and interpretation. University of Minnesota Press.

Blais, J., Knack, N., Baglole, J.S., \& Stevenson, A. (2021). Data and syntax for Susceptibility of the Self-Report Psychopathy Scale (SRP 4) to response distortion and the utility of including validity indices to detect deception [Data set]. https://osf.io/gau8f/

Boduszek, D., \& Debowska, A. (2016). Critical evaluation of psychopathy measurement (PCL-R and SRP-III/SF) and recommendations for future research. Journal of Criminal Justice, 44, 1-12. https://doi.org/10.1016/j.jcrimjus.2015.11.004

Book, A., Holden, R., Starzyk, K., Wasylkiw, L., \& Edwards, M. (2006). Psychopathic traits and experimentally induced deception in self-report assessment. Personality and Individual Differences, 41(4), 601-608. https://doi.org/10.1016/j.paid.2006.02.011

Brinkley, C. A., Diamond, P. M., Magaletta, P. R., \& Heigel, C. P. (2008). Cross-validation of Levenson's psychopathy scale in a sample of federal female inmates. Assessment, 15(4), 464-482. https://doi.org/10.1177/1073191108319043 
Cleckley, H. M. (1976). The mask of sanity (5th ed.). Mosby.

Declercq, F., Carter, R., \& Neumann, C. S. (2015). Assessing psychopathic traits and criminal behavior in a young adult female community sample using the self-report psychopathy scale. Journal of Forensic Sciences, 60(4), 928-935. https://doi.org/10.1111/15564029.12783

deVries, R. E., Zettler, I., \& Hilbig, B. E. (2014). Rethinking trait conceptions of social desirability scales: Impression management as an expression of honesty-humility. Assessment, 21, 286-299. 10.1177/1073191113504619

Dotterer, H. L., Waller, R., Neumann, C. S., Shaw, D. S., Forbes, E. E., Hariri, A. R., \& Hyde, L. W. (2017). Examining the factor structure of the self-report of psychopathy short-form across four young adult samples. Assessment, 24(8), 1062-1079. https://doi.org/10.1177/ 1073191116640355

Edens, J., Buffington, J., \& Tomicic, T. (2000). An Investigation of the Relationship between Psychopathic Traits and Malingering on the Psychopathic Personality Inventory. Assessment, 7(3), 281-296. https://doi.org/10.1177/107319110000700307

Edens, J., Buffington, J., Tomicic, T., \& Riley, B. (2001). Effects of positive impression management on the psychopathic personality inventory. Law and Human Behavior, 25(3), 235-256. https://doi.org/10.1023/A:1010793810896

Faul, F., Erdfelder, E., Lang, A. G., \& Buchner, A. (2007). G*Power 3: A flexible statistical power analysis program for the social, behavioral, and biomedical sciences. Behavior Research Methods, 39, 175-191. https://doi.org/10.3758/BF03193146

Furnham, A. (1986). Response bias, social desirability and dissimulation. Personality and Individual Differences, 7(3), 385-400. https://doi.org/10.1016/0191-8869(86)90014-0 
Furnham, A., \& Henderson, M. (1982). The good, the bad and the mad: Response bias in selfreport measures. Personality and Individual Differences, 3(3), 311320. https://doi.org/10.1016/0191-8869(82)90051-4

Gillard, N. D., \& Rogers, R. (2015). Denial of risk: The effects of positive impression management on risk assessments for psychopathic and nonpsychopathic offenders. International Journal of Law and Psychiatry, 42-43, 106-113. https://doi.org/10.1016/j.ijlp.2015.08.014

Gordts, S., Uzieblo, K., Neumann, C., Van Den Bussche, E., \& Rossi, G. (2017). Validity of the Self-Report Psychopathy scales (SRP-III full and short versions) in a community sample. Assessment, 24(3), 308-325. https://doi.org/10.1177/1073191115606205

Hare, R. D. (2003). Manual for the Hare Psychopathy Checklist-Revised (2nd ed.). Multi-Health Systems.

Holden, R. R. (2008). Underestimating the effects of faking on the validity of self-report personality scales. Personality and Individual Differences, 44(1), 311-321. https://doi.org/10.1016/j.paid.2007.08.012

Hough, L. M. (1998). Effects of intentional distortion in personality measurement and evaluation of suggested palliatives. Human Performance, 11(2-3), 209244. https://doi.org/10.1207/s15327043hup1102\&3_6

Kelsey, K. R., Rogers, R., \& Robinson, E. V. (2015). Self-report measures of psychopathy: What is their role in forensic assessments? Journal of Psychopathology and Behavioral Assessment, 37(3), 380-391. https://doi.org/10.1007/s10862-014-9475-5 
Levenson, M. R., Kiehl, K. A., \& Fitzpatrick, C. M. (1995). Assessing psychopathic attributes in a noninstitutionalized population. Journal of Personality and Social Psychology, 68(1), 151-158. https://doi.org/10.1037/0022-3514.68.1.151.

Lilienfeld, S. O., \& Widows, M. (2005). Manual for the Psychopathic Personality InventoryRevised (PPI-R). Psychological Assessment Resources.

Luhtanen, R. K., \& Crocker, J. (2005). Alcohol use in college students: Effects of level of selfesteem, narcissism, and contingencies of self-worth. Psychology of Addictive Behaviors, 19(1), 99-103. https://doi.org/10.1037/0893-164X.19.1.99

MacNeil, B., \& Holden, R. (2006). Psychopathy and the detection of faking on self-report inventories of personality. Personality and Individual Differences, 41(4), 641-651. https://doi.org/10.1016/j.paid.2006.03.004

Mahmut, M. K., Menictas, C., Stevenson, R. J., \& Homewood, J. (2011). Validating the factor structure of the self-report psychopathy scale in a community sample. Psychological Assessment, 23(3), 670-678. https://doi.org/10.1037/a0023090

Marion, B. E., Sellbom, M., Salekin, R. T., Toomey, J. A., Kucharski, L. T., \& Duncan, S. (2013). An examination of the association between psychopathy and dissimulation using the MMPI-2-RF validity scales. Law and Human Behavior, 37(4), 219-230. https://doi.org/10.1037/lhb0000008

McGrath, R. E., Mitchell, M., Kim, B. H., \& Hough, L. (2010). Evidence for response bias as a source of error variance in applied assessment. Psychological Bulletin, 136, 450 - 470. http://dx.doi.org/10.1037/a0019216 
Miller, J. D., Jones, S. E., \& Lynam, D. R. (2011). Psychopathic traits from the perspective of self and informant reports: Is there evidence for a lack of insight? Journal of Abnormal Psychology, 120, 758 -764. http://dx.doi.org/10.1037/a0022477

Neumann, C. S., Hare, R. D., \& Pardini, D. A. (2015). Antisociality and the construct of psychopathy: Data from across the globe. Journal of Personality, 83(6), 678-692. https://doi.org/10.1111/jopy.12127

Neumann, C., \& Pardini, D. (2014). Factor structure and construct validity of the Self-Report Psychopathy (SRP) scale and the Youth Psychopathic Traits Inventory (YPI) in young men. Journal of Personality Disorders, 28(3), 419-33. https://doi.org/10.1521/pedi_2012_26_063

Newman, D.A. (2014). Missing data: Five practical guidelines. Organizational Research Methods, 17(4), 372-411. https://doi.org/10.1177/1094428114548590

Parent, M.C. (2013). Handling item-level missing data: Simpler is just as good. The Counseling Psychologist, 41(4), 568-600. https://doi.org/10.1177/0011000012445176

Paulhus, D. L., Neumann, C. S., \& Hare, R. D. (2017). The SRP 4: Self-report psychopathy scale (4th ed.). Multi-Health Systems.

Ray, J. V., Hall, J., Rivera-Hudson, N., Poythress, N. G., Lilienfeld, S. O., \& Morano, M. (2013). The relation between self-reported psychopathic traits and distorted response styles: A meta-analytic review. Personality Disorders, 4(1), 1-14. https://doi.org/10.1037/a0026482

Reidy, D. E., Kearns, M. C., DeGue, S., Lilienfeld, S. O., Massetti, G., \& Kiehl, K. A. (2015). Why psychopathy matters: Implications for public health and violence prevention. Aggression and Violent Behavior, 24, 214-225. https://doi.org/10.1016/j.avb.2015.05.018 
Rogers, R., Vitacco, M. J., Jackson, R. L., Martin, M., Collins, M., \& Sewell, K. W. (2002). Faking psychopathy? An examination of response styles with antisocial youth. Journal of Personality Assessment, 78(1), 31-46. https://doi.org/10.1207/S15327752JPA7801_0

Rohling, M. L., Larrabee, G. J, Greiffenstein, M. F., Ben-Porath, Y. S., Lees-Haley, P., Green, P., \& Greve, K. W. (2011) A misleading review of response bias: Comment on McGrath, Mitchell, Kim, \& Hough (2010). Psychological Bulletin, 137(4), 708-712. https://doi.org/10.1037/a0023327

Salekin, R. T., Chen, D. R., Sellbom, M., Lester, W. S., \& MacDougall, E. (2014). Examining the factor structure and convergent and discriminant validity of the Levenson self-report psychopathy scale: Is the two-factor model the best fitting model? Personality Disorders: Theory, Research, and Treatment, 5(3), 289-304. https://doi.org/10.1037/per0000073

Sellbom, M. (2011). Elaborating on the construct validity of the Levenson self-report psychopathy scale in incarcerated and nonincarcerated samples. Law and Human Behavior, 35(6), 440-451. https://doi.org/10.1007/s10979-010-9249-x

Sellbom, M. (2017). Using the MMPI-2-RF to characterize defendants evaluated for competency to stand trial and criminal responsibility. International Journal of Forensic Mental Health, 16(4), 304-312. https://doi.org/10.1080/14999013.2017.1371259

Sellbom, M., Lilienfeld, S. O., Fowler, K. A., \& McCrary, K. L. (2018). The self-report assessment of psychopathy: Challenges, pitfalls, and promises. In C. J. Patrick (Ed.), Handbook of psychopathy (2nd ed; pp. 211-258). The Guilford Press.

Uziel, L. (2010). Rethinking social desirability scales: From impression management to interpersonally oriented self-control. Perspectives on Psychological Science, 5, 243-262. https://www.jstor.org/stable/41613334 
Verschuere, B., \& te Kaat, L. (2020). What are the core features of psychopathy? A prototypicality analysis using the psychopathy checklist-revised (PCL-R). Journal of Personality Disorders, 34(3), 410-419. https://doi.org/10.1521/pedi_2019_33_396

Vitacco, M. J., Neumann, C. S., \& Jackson, R. (2005). Testing a four-factor model of psychopathy and its association with ethnicity, gender, intelligence, and violence. Journal of Consulting and Clinical Psychology, 73(3), 466-476. https://doi.org/10.1037/0022006X.73.3.466

Wang, M.-C., Shou, Y., Deng, Q., Sellbom, M., Salekin, R. T., \& Gao, Y. (2018). Factor structure and construct validity of the Levenson self-report psychopathy scale (LSRP) in a sample of Chinese male inmates. Psychological Assessment, 30(7), 882-892. https://doi.org/10.1037/pas0000537.

Watts, A. L., Lilienfeld, S. O., Edens, J. F., Douglas, K. S., Skeem, J. L., Verschuere, B., \& LoPilato, A. C. (2016). Does response distortion statistically affect the relations between self-report psychopathy measures and external criteria? Psychological Assessment, 28(3), 294-306. https://doi.org/10.1037/pas0000168

Williams, K. M., Paulhus, D. L., \& Hare, R. D. (2007). Capturing the four-factor structure of psychopathy in college students via self-report. Journal of Personality Assessment, 88(2), 205-219.

Zwets, A. J., Hornsveld, R. H. J., Neumann, C. S., Muris, P., \& Van Marle, H. J. C. (2015). The four-factor model of the psychopathy checklist-revised in Dutch forensic inpatients with a personality disorder and forensic inpatients with a chronic psychotic disorder. International Journal of Law and Psychiatry, 39, 13-22. https://doi:10.1016/j.ijlp.2015.01.016 


\section{Figure 1}

Significant Interaction between Condition and Time for SRP Total Scores

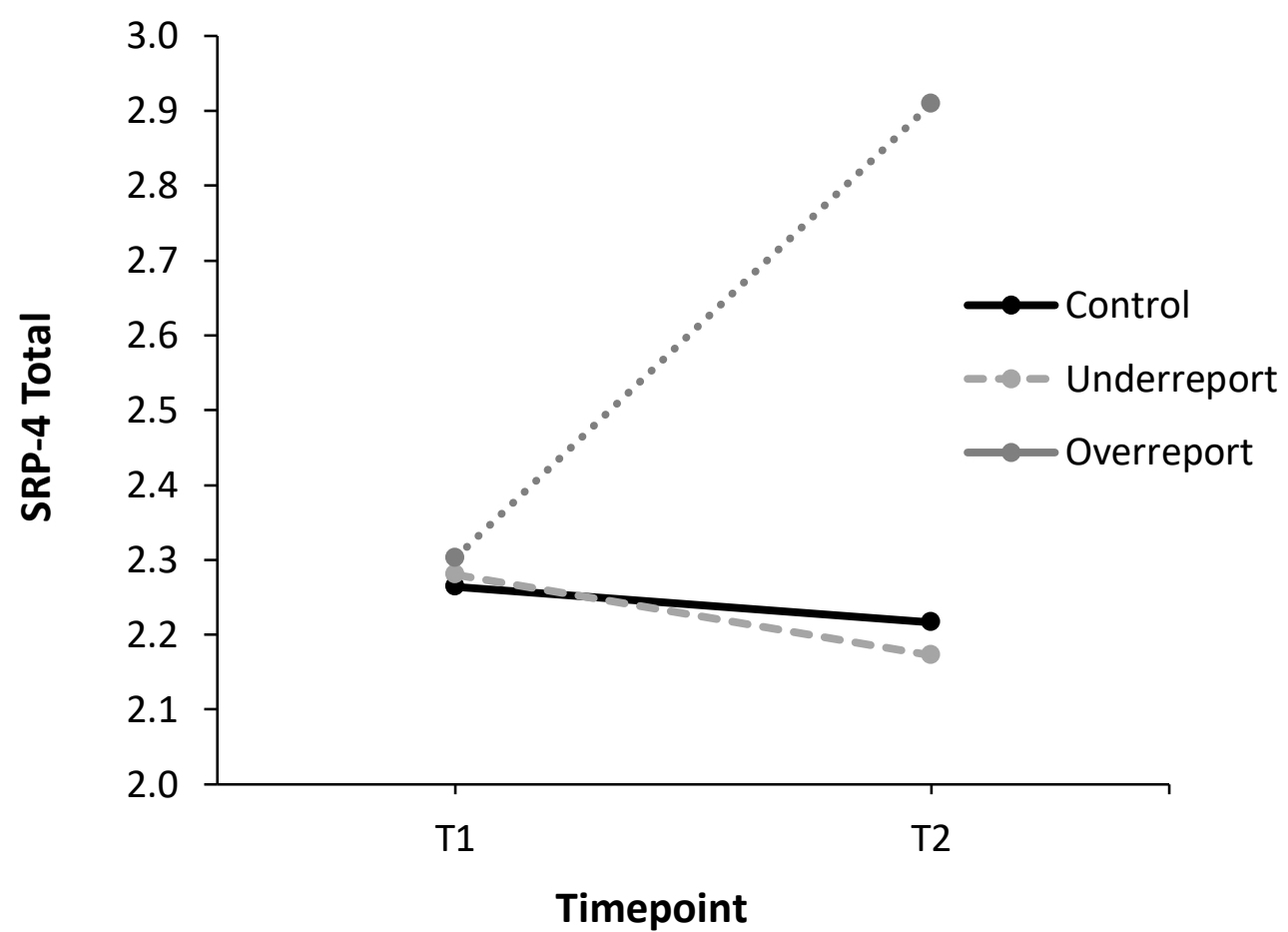

Note. SRP $=$ Self-Report Psychopathy Scale $\left(4^{\text {th }}\right.$ version $) . \mathrm{T} 1=$ Time 1 (pre-scenario). T2 $=$ Time 2 (post-scenario). Range of possible scores $=1$ to 5 . Interaction plots for all SRP facet scores are similar to the plot above (see Appendix B in online supplemental materials). 


\section{Table 1}

Mean SRP Total and Facet Scores at T1 and T2

\begin{tabular}{|c|c|c|c|c|c|c|c|c|c|}
\hline \multirow{2}{*}{$\begin{array}{l}\text { SRP } \\
\text { Scale }\end{array}$} & \multirow{2}{*}{ Control } & \multirow{2}{*}{$\begin{array}{l}\text { Under } \\
M(S D)\end{array}$} & \multirow[t]{2}{*}{ Over } & \multicolumn{3}{|c|}{ Control vs. Under } & \multicolumn{3}{|c|}{ Control vs. Over } \\
\hline & & & & $t$ & $p$ & $\begin{array}{l}\text { Cohen's } d \\
{[95 \% \mathrm{CI}]}\end{array}$ & $t$ & $p$ & $\begin{array}{l}\text { Cohen's } d \\
{[95 \% \mathrm{CI}]}\end{array}$ \\
\hline \multicolumn{10}{|c|}{ Interpersonal Manipulation } \\
\hline $\mathrm{T} 1$ & $\begin{array}{c}2.42 \\
(0.59)\end{array}$ & $\begin{array}{c}2.40 \\
(0.61)\end{array}$ & $\begin{array}{c}2.38 \\
(0.59)\end{array}$ & 0.15 & .879 & $0.02[-0.22,0.27]$ & -0.45 & .653 & $0.07[-0.18,0.31]$ \\
\hline $\mathrm{T} 2$ & $\begin{array}{l}2.32^{* *} \\
(0.64)\end{array}$ & $\begin{array}{l}2.22^{* *} \\
(0.62)\end{array}$ & $\begin{array}{l}2.99^{* *} \\
(0.96)\end{array}$ & 1.11 & .267 & $0.15[-0.10,0.39]$ & 7.88 & $<.0001$ & $0.82[0.57,1.08]$ \\
\hline \multicolumn{10}{|c|}{ Callous Affect } \\
\hline $\mathrm{T} 1$ & $\begin{array}{c}2.54 \\
(0.34)\end{array}$ & $\begin{array}{c}2.53 \\
(0.40)\end{array}$ & $\begin{array}{c}2.54 \\
(0.42)\end{array}$ & 0.07 & .947 & $0.01[-0.23,0.25]$ & 0.002 & .999 & $0.00[-0.24,0.25]$ \\
\hline $\mathrm{T} 2$ & $\begin{array}{c}2.53 \\
(0.35)\end{array}$ & $\begin{array}{c}2.49 \\
(0.40)\end{array}$ & $\begin{array}{l}2.98^{\text {** }} \\
(0.63)\end{array}$ & 0.72 & .470 & $0.10[-0.14,0.35]$ & 8.47 & $<.0001$ & $0.91[0.65,1.16]$ \\
\hline \multicolumn{10}{|c|}{ Erratic Lifestyle } \\
\hline $\mathrm{T} 1$ & $\begin{array}{c}2.39 \\
(0.65)\end{array}$ & $\begin{array}{c}2.44 \\
(0.65)\end{array}$ & $\begin{array}{c}2.52 \\
(0.64)\end{array}$ & 0.55 & .586 & $0.07[-0.17,0.32]$ & 1.51 & .133 & $0.21[-0.04,0.45]$ \\
\hline $\mathrm{T} 2$ & $\begin{array}{c}2.36 \\
(0.67)\end{array}$ & $\begin{array}{c}2.28^{*} \\
(0.69)\end{array}$ & $\begin{array}{l}3.10^{\text {** }} \\
(0.94)\end{array}$ & 0.85 & .396 & $0.11[-0.13,0.36]$ & 8.36 & $<.0001$ & $0.92[0.66,1.17]$ \\
\hline \multicolumn{10}{|c|}{ Antisocial Behaviour } \\
\hline $\mathrm{T} 1$ & $\begin{array}{c}1.71 \\
(0.55)\end{array}$ & $\begin{array}{c}1.75 \\
(0.62)\end{array}$ & $\begin{array}{c}1.77 \\
(0.60)\end{array}$ & 0.38 & .704 & $0.06[-0.19,0.30]$ & 0.68 & .495 & $0.10[-0.15,0.35]$ \\
\hline $\mathrm{T} 2$ & $\begin{array}{c}1.67 \\
(0.56)\end{array}$ & $\begin{array}{c}1.70 \\
(0.59)\end{array}$ & $\begin{array}{l}2.57^{* *} \\
(1.03)\end{array}$ & 0.38 & .703 & $0.06[-0.19,0.30]$ & 10.70 & $<.0001$ & $1.10[0.84,1.36]$ \\
\hline \multicolumn{10}{|c|}{ SRP Total } \\
\hline $\mathrm{T} 1$ & $\begin{array}{c}2.26 \\
(0.44)\end{array}$ & $\begin{array}{c}2.28 \\
(0.47)\end{array}$ & $\begin{array}{c}2.30 \\
(0.45)\end{array}$ & 0.24 & .451 & $0.04[-0.21,0.28]$ & 0.58 & .563 & $0.09[-0.16,0.33]$ \\
\hline $\mathrm{T} 2$ & $\begin{array}{l}2.22^{*} \\
(0.45)\end{array}$ & $\begin{array}{c}2.17^{*} \\
(0.49)\end{array}$ & $\begin{array}{l}2.91^{\text {** }} \\
(0.83)\end{array}$ & 0.66 & .468 & $0.09[-0.15,0.34]$ & 10.39 & $<.0001$ & $1.05[0.79,1.31]$ \\
\hline
\end{tabular}

Note. SRP $=$ Self-Report Psychopathy Scale $\left(4^{\text {th }}\right.$ version $)$. T1 $=$ Time 1. T2 $=$ Time 2 . Range of possible scores $=1$ to 5.

* For T1 vs. T2, $p<.001$. ** For T1 vs. T2, $p \leq .0001$. 


\section{Table 2}

Correlation Magnitudes for Scores on LSRP at T1 and SRP at T2

\begin{tabular}{|c|c|c|c|c|c|c|c|c|}
\hline \multirow{2}{*}{$\begin{array}{l}\text { LSRP Scale } \\
\quad(\mathrm{T} 1)\end{array}$} & \multirow{2}{*}{$\begin{array}{l}\text { SRP Scale } \\
\quad \text { (T2) }\end{array}$} & \multirow[b]{2}{*}{ Control } & \multirow[b]{2}{*}{ Under } & \multirow[b]{2}{*}{ Over } & \multicolumn{2}{|c|}{ Control vs. Under } & \multicolumn{2}{|c|}{ Control vs. Over } \\
\hline & & & & & $z$ & $\begin{array}{l}\text { Cohen's } q \\
{[95 \% \mathrm{CI}]}\end{array}$ & $z$ & $\begin{array}{l}\text { Cohen's } q \\
{[95 \% \mathrm{CI}]}\end{array}$ \\
\hline $\begin{array}{l}\text { Egocentricity } \\
\qquad(\mathrm{F} 1)\end{array}$ & $\begin{array}{l}\text { Interpersonal } \\
\text { Manipulation }\end{array}$ & .67 & .53 & .05 & 1.75 & $0.22[-0.03,0.47]$ & $6.02^{*}$ & $0.76[0.51,1.01]$ \\
\hline $\begin{array}{c}\text { Callousness } \\
\text { (F2) }\end{array}$ & $\begin{array}{c}\text { Callous } \\
\text { Affect }\end{array}$ & .36 & .41 & -.001 & 0.47 & $0.06[-0.19,0.31]$ & $2.99^{*}$ & $0.38[0.13,0.63]$ \\
\hline $\begin{array}{c}\text { Antisociality } \\
\text { (F3) }\end{array}$ & $\begin{array}{c}\text { Erratic } \\
\text { Lifestyle }\end{array}$ & .65 & .55 & .08 & 1.25 & $0.16[-0.09,0.40]$ & $5.50^{*}$ & $0.70[0.45,0.95]$ \\
\hline $\begin{array}{c}\text { Antisociality } \\
\text { (F3) }\end{array}$ & $\begin{array}{l}\text { Antisocial } \\
\text { Behaviour }\end{array}$ & .44 & .37 & -.05 & 0.67 & $0.08[-0.16,0.33]$ & $4.14^{*}$ & $0.52[0.27,0.77]$ \\
\hline LSRP Total & SRP Total & .70 & .63 & .01 & 1.00 & $0.13[-0.12,0.37]$ & $6.79^{*}$ & $0.86[0.61,1.11]$ \\
\hline
\end{tabular}

Note. Using Pearson's $r$ correlations. LSRP = Levenson Self-Report Psychopathy Scale. SRP $=$ Self-Report Psychopathy Scale $\left(4^{\text {th }}\right.$ version). Since the LSRP and SRP have different factor structures, we correlated LSRP F3 with both Facets 3 and 4 on the SRP.

$* z>1.96$ is significant at $p<.05$. 


\section{Table 3}

Differences in Mean VR and DR Scores between Conditions

\begin{tabular}{|c|c|c|c|c|c|c|c|c|c|}
\hline \multirow{2}{*}{$\begin{array}{l}\text { Validity } \\
\text { Scale } \\
\text { (T2) }\end{array}$} & Control & Clinical & Under & Over & \multirow[b]{2}{*}{$F$} & \multirow[b]{2}{*}{$p$} & $\begin{array}{l}\text { Control vs. } \\
\text { Under }\end{array}$ & $\begin{array}{l}\text { Control vs. } \\
\text { Over }\end{array}$ & $\begin{array}{l}\text { Clinical vs. } \\
\text { Over }\end{array}$ \\
\hline & \multicolumn{4}{|c|}{$M(S D)$} & & & \multicolumn{3}{|c|}{ Cohen's $d[95 \% \mathrm{CI}]$} \\
\hline VR & $\begin{array}{c}2.40 \\
(0.47)\end{array}$ & $\begin{array}{c}2.40 \\
(0.54)\end{array}$ & $\begin{array}{c}2.50 \\
(0.51)\end{array}$ & $\begin{array}{c}2.17 \\
(0.58)\end{array}$ & 9.20 & $<.0001$ & $\begin{array}{c}0.22 \\
{[-0.03,0.46]}\end{array}$ & $\begin{array}{c}0.44^{*} \\
{[0.19,0.68]}\end{array}$ & $\begin{array}{c}0.41^{*} \\
{[0.15,0.67]}\end{array}$ \\
\hline $\mathrm{DR}$ & $\begin{array}{c}1.37 \\
(0.32)\end{array}$ & $\begin{array}{c}1.26 \\
(0.30)\end{array}$ & $\begin{array}{c}1.40 \\
(0.41)\end{array}$ & $\begin{array}{c}2.01 \\
(0.79)\end{array}$ & 54.04 & $<.0001$ & $\begin{array}{c}0.09 \\
{[-0.15,0.34]}\end{array}$ & $\begin{array}{c}1.07^{* *} \\
{[0.82,1.31]}\end{array}$ & $\begin{array}{c}1.19^{* *} \\
{[0.93,1.46]}\end{array}$ \\
\hline
\end{tabular}

Note. VR = Virtuous Responding subscale from PPI-R. DR = Deviant Responding subscale from PPI-R. Range of possible scores $=1$ to 4 .

$* p<.01 . * * p<.0001$. 